\title{
Stop of loss of cognitive performance during rehabilitation after total hip arthroplasty—Prospective controlled study
}

\author{
Matthias H. Brem, MD, MHBA $;^{1 *}$ Siegfried Lehrl, PhD $;^{2-3}$ Anna K. Rein, MD ${ }^{2}$ Sylvia Massute, BS; ${ }^{2}$ Stefan

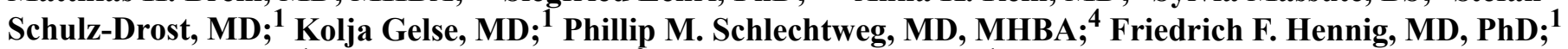 \\ Alexander Olk, MD; ${ }^{1}$ Jürgen H. Jacob, MD; ${ }^{2}$ Johannes Gusinde, MD $^{1}$ \\ Departments of ${ }^{1}$ Surgery, Division of Orthopaedic and Trauma Surgery, and ${ }^{2}$ Psychiatry and Psychotherapy, \\ Friedrich-Alexander Universität Erlangen-Nürnberg, Erlangen, Germany; ${ }^{3}$ Competence and Research Centre for Bio- \\ mental Promotion of Education and Health, Academy of Health, Mainburg, Germany; ${ }^{4}$ Department of Radiology, \\ Friedrich-Alexander Universität Erlangen-Nürnberg, Erlangen, Germany
}

\begin{abstract}
Prolonged hospitalization is known to be associated with a loss of cognitive performance. Does playing video games (VGs) developed to improve cognitive properties delay this loss or even lead to an increase in cognitive performance? We performed a 10-day longitudinal study of patients who received total hip arthroplasty. We compared 16 patients (6 male) aged $66 \pm 9$ years (mean \pm standard deviation) who played $D r$. Kawashima's Brain Training: How Old Is Your Brain? (Nintendo; Redmond, Washington) on a Nintendo DS handheld console with 16 control patients (6 male) aged $69 \pm 14$ years. We measured cognitive performance 1 day preoperation, as well as on days 2 and 9 postoperation. With the daily exercise of a specific VG by the play group, the patients' fluid intelligence (median intelligence quotient 99-106), working memory capacity, and rate of information processing significantly improved over the course of 7 postoperative days. The cognitive performance of the control group did not increase. However, the memory spans of both groups did not systematically change. Exercise with VGs can prevent the loss of cognitive performance during prolonged hospitalization.
\end{abstract}

Key words: cognitive performance, crystallized intelligence, fluid intelligence, hospitalization, intelligence quotient, rate of information processing, rehabilitation, total hip arthroplasty, video game, working memory capacity.

\section{INTRODUCTION}

In 1988, Billig et al. presented the results of a longitudinal study of patients hospitalized with hip fracture [1]. In this study, 40 percent of patients developed a loss of cognitive performance. Similar results may be expected of many other patients during prolonged hospitalization following surgical interventions, e.g., total hip arthroplasty (THA).

Recently, a number of available games have claimed to improve, or at least maintain, cognitive performance. In particular, video games (VGs) using a personal computer, game console, or handheld game console can be

\footnotetext{
Abbreviations: $\mathrm{IQ}=$ intelligence quotient, $\mathrm{SD}=$ standard deviation, $\mathrm{TA}=$ time point of assessment, $\mathrm{THA}=$ total hip arthroplasty, VG = video game.

*Address all correspondence to Matthias H. Brem, MD, MHBA; Department of Surgery, Division of Orthopaedic and Trauma Surgery, Friedrich-Alexander Universität Erlangen-Nürnberg, Krankenhausstr 12, D-91054 Erlangen, Germany; +49-9131-8542119; fax: +49-9131-8533300. Email: brem@bwh.harvard.edu

DOI:10.1682/JRRD.2010.01.0011
} 
played without a partner and, thus, may be suitable for hospitalized patients.

Such VGs have been described to effectively improve the cognitive performance and surgical skills of young surgeons [2]. Rosser et al. reported that physicians reduced the time of surgical interventions by 27 percent and the rate of mistakes by 37 percent after using specific VGs [2].

We evaluated whether the VG Dr. Kawashima's Brain Training: How Old Is Your Brain? (Nintendo; Redmond, Washington), which was developed particularly for cerebral and cognitive activation, played on the Nintendo DS handheld game console (Nintendo) may help circumvent the loss of or even improve cognitive performance during hospitalization and rehabilitation [3].

\section{METHODS}

\section{Patients and Study Design}

This study was a prospective nonblinded controlled longitudinal study of patients who underwent THA. Inclusion criteria comprised patients aged 45 years (no maximum) with full contractual capability. As much as possible, we avoided using patients on medication for analgesic therapy, which can dramatically modulate mental performance. All patients received the same regimen of postoperative painkilling drugs consisting of Valorin (naloxon + tilidin) and Novalgin (metamizol).

We compared 16 patients (play group) who played Dr. Kawashima's Brain Training: How Old Is Your Brain? (in-game exercises of their choice) for 30 minutes daily with 16 patients (control group) who did not play the VG. We randomly assigned the patients to either the play group or the control group. All patients agreed to participate in either group before they enrolled in the study. We evaluated all patients before enrollment concerning their social and physical activity statuses. Before the surgical intervention (THA), we evaluated physical activity status by using the Harris Hip Score and the Merle d'Aubigne score. We only included patients with similar physical activity statuses to reduce confounding variables. The two groups did not differ in their social statuses prior to the surgery.

The patients in the play group and control group were $66.1 \pm 9.3$ and $68.9 \pm 13.9$ years old (all values mean \pm standard deviation [SD] unless otherwise specified), respectively (Table). Since both groups included 6 male and 10 female patients, the groups were comparable with respect to age and sex (Table). None of the patients had any experience with the Nintendo DS or Dr. Kawashima's Brain Training: How Old Is Your Brain? prior to the study.

According to the statistical analysis (Table), the play group had significantly higher preoperative levels of crystallized and fluid intelligence and working memory capacity. However, we found no significant preoperative differences $(p=0.31)$ in the rate of information processing. Therefore, the initial differences in working memory capacity and fluid intelligence can be ascribed to the initial differences in the length of memory span between the groups.

\section{Variables and Course of Investigation}

At the time of admission, we recorded the patients' sex, age, and body mass index, as well as diseases such as osteoarthritis. We labeled the day before surgery as time point of assessment (TA) 1. Multiple Choice Vocabulary Test-B served to determine the initial level of intelligence quotient (IQ) [3]. This test measures the IQ of crystallized intelligence, which reaches its maximum at 20 years of age and changes little during adulthood. Furthermore, crystallized intelligence is, to a large extent, independent of psychogenic disorders. Therefore, during periods of psychogenic disorders it assesses the level of premorbid IQ.

We scheduled TA2 for postoperative day 2. On that day, patients resumed the VG exercises and played daily until discharge, with the time set by each patient ( $\geq 35 \mathrm{~min}$ ). We scheduled TA3 for postoperative day 9 (7 days following TA2).

We measured and documented the following parameters at each TA:

- We used the Kurztest fur Allgemeine Intelligenz, a short test for basic parameters of central information processing, to measure the rate of information processing (bits per second) and the length of memory span (seconds or chunks [i.e., units in mind such as letters, digits, or one-syllable words]) [4-5]. This test assesses working memory capacity (bit), which is the product of the two basic parameters of central information processing. In addition, it correlates with the level of fluid intelligence.

- We used the 12-Item Short Form Health Survey to measure health-related quality of life [6].

- We used the Neuroticism-Extroversion-OpennessFive Factor Inventory to measure mental stability and 
Table.

Age and parameters of cognitive performance in 32 total hip arthroplasty patients: 16 who played video games ("play”) and 16 controls.

\begin{tabular}{|c|c|c|c|c|c|c|}
\hline Variable & Median & Mean \pm SD & Minimum & Maximum & $p$-Value ${ }^{*}$ & $p$-Value \\
\hline \multicolumn{7}{|l|}{$\overline{\text { Age (years) }}$} \\
\hline Control & 66.0 & $68.9 \pm 13.9$ & 44 & 86 & - & - \\
\hline \multicolumn{7}{|l|}{ Crystallized IQ } \\
\hline Control & 107.0 & $106.4 \pm 14.7$ & 85 & 136 & - & - \\
\hline Play & 118.0 & $119.5 \pm 13.8$ & 91 & 143 & $0.02^{\ddagger}$ & - \\
\hline Capacity (bits) & & & & & & \\
\hline \multicolumn{7}{|l|}{ TA1 } \\
\hline Control & 63.0 & $66.1 \pm 30.0$ & 11.7 & 121.3 & - & - \\
\hline Play & 88.0 & $87.4 \pm 23.0$ & 51.0 & 123.7 & $0.03^{\ddagger}$ & - \\
\hline \multicolumn{7}{|l|}{ TA2 } \\
\hline Play & 90.3 & $92.2 \pm 25.4$ & 52.0 & 146.6 & $0.004^{\S}$ & 0.004 \\
\hline \multicolumn{7}{|c|}{$\begin{array}{l}\text { Difference } \\
\text { (TA3 - TA2) }\end{array}$} \\
\hline Control & 0.9 & $2.8 \pm 15.1$ & -21.2 & 33.4 & - & - \\
\hline Play & 8.6 & $9.9 \pm 20.3$ & -21.8 & 60.8 & $0.19^{\S}$ & - \\
\hline
\end{tabular}

*Significance between groups.

${ }^{\dagger}$ Significance of change (TA3 vs TA2) within group.

‡One-sided.

§Two-sided.

$\mathrm{IQ}=$ intelligence quotient, $\mathrm{SD}$ = standard deviation, TA1 = time point of assessment 1 day preoperation, TA2 = time point of assessment 2 days postoperation, TA3 = time point of assessment 9 days postoperation.

extraversion (both parameters provide information on the ability to communicate) [7].

- We used Patient-Reported Outcomes Scales to measure whether the patient was mentally excessive or lacking in demands during hospitalization [8].

- We also judged whether the patient was currently in an uncomfortable mood (including pain).

\section{Nine Different Exercises}

Following TA1, we explained the Nintendo DS and VG and the play group played for the first time. We took nine of the exercises in Dr. Kawashima's Brain Training: How Old Is Your Brain? into consideration, among which one or more had to be accomplished in each session. The time frame for every patient was to play $\geq 35$ min per day.

Exercise examples include-

- Mental calculation: The player must solve 20 arithmetic problems as fast as possible by entering the results on the touch screen of the Nintendo DS.
- Reading: The player must read a sample of a conventional text out loud as fast as possible. The VG measures the time until finished and calculates the number of syllables per second.

- Memory: The player must review a series of numbers displayed for $1 \mathrm{~s}$, then tap their position on the touch screen in the correct increasing order.

- Working memory: The player must count the number of persons leaving or entering a house, then specify the number of persons in the house at the end of the exercise.

- Spoken calculation: The player must speak the results of 50 simple arithmetic problems into the microphone of the Nintendo DS.

\section{Specific Assumptions}

Nurses ensured that patients in the play group used the VG once or twice between TA1 and TA2 and on each day between TA2 and TA3. Jaeggi et al. proved that the 
longer the training for working memory capacity, the higher the gain in fluid intelligence [9]. Therefore, we could expect the most prominent changes between TA2 and TA3, provided that playing VGs influences mental performance.

This allowed us to formulate two hypotheses for the play group:

1. The working memory capacity that consists of both measured basic parameters of information processing (rate of information processing and length of memory span) will increase between TA2 and TA3. If this hypothesis is affirmed, the same change applies to the fluid IQ, which is based on the assignment of the working memory capacity to a Gaussian distribution of mental performance [4-5].

2. The play group will achieve better results in working memory capacity than the control group at TA3.

\section{Statistics}

We used nonparametric tests for statistical analysis. We also used parametric arithmetic (mean \pm SD) for statistical description. We used nonparametric methods for inference statistical analysis and correlations (Spearman $\rho$ ), because they make fewer assumptions about the level of measurement and distribution of data than parametric statistics. For statistical description besides the nonparametric median, we additionally administered the parametric mean \pm SD since it is more often used in international publications.

\section{RESULTS}

The play group adhered to the VG regimen for a minimum of $\geq 35$ min per day under the supervision of a study nurse. Durations of training varied between 35 and 70 min per day. The average play time was $45 \mathrm{~min}$ a day.

Working memory capacity significantly increased from 78.2 to 90.3 bits $(p=0.004)$ between TA2 and TA3, confirming the first hypothesis (Table). Analogously, fluid intelligence increased significantly from 99 to 106 $(p=0.006)$ (Figure 1).

Is the increase in the capacity of working memory due to both basic parameters of information processing or to only one? The median of the rate of information processing for the play group increased significantly from 13.5 to $14.9 \mathrm{bit} / \mathrm{s}$ during this period $(p=0.03)$ (Figure 2), whereas the rise in length of memory span (Figure 3), which increased from 5.6 to 6.0 chunks, did not reach the level of significance ( $p=0.334)$. Therefore, the increase in working memory capacity depended more on rate of information processing and less on length of memory span. In comparison, the control group showed only minor improvements; none reached the level of significance (Figures 1-3, Table).

The working memory capacity of the play group was significantly higher than that of the control group at TA3 ( $p=0.004$ ), thus confirming the second hypothesis. At TA3, the differences in its components were significant, too (rate of information processing: $p=0.03$; length of memory span: $p=0.002$ ). However, we must consider that significant differences were already noticeable at TA2 between the two groups in all variables except for the rate of information processing (Table, Figures 1-3). From TA2 to TA3, the distance between medians of the cognitive variables increased between the control group and the play group, favoring the latter. The comparisons of the differences of changes between both groups did not reach significance; however, working memory capacity ( $p=0.19)$ was followed by the rate of information processing $(p=0.24)$ and memory span $(p=0.48)$. Therefore, the main contribution to the change of working memory capacity that confirms the second hypothesis derived from the rate of information processing and not from memory span.

Finally, we have examined whether individuals with different levels of mental efficiency have the same capacity for change. Within the play group, we chose the working

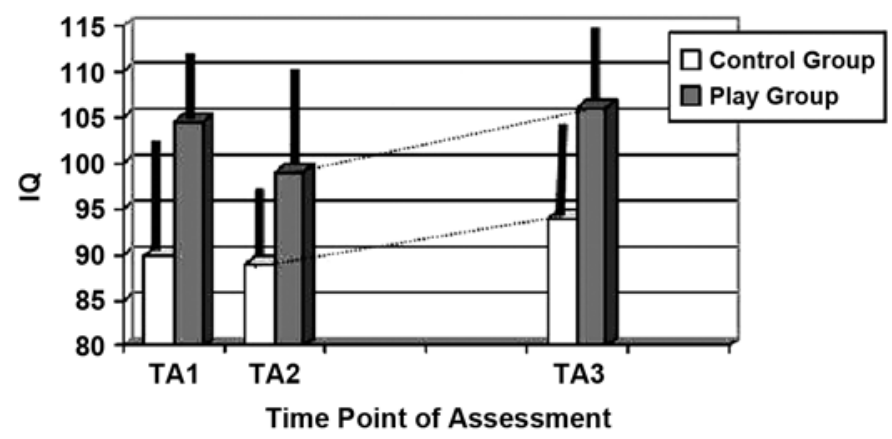

Figure 1.

Median and mean quartile of fluid intelligence quotient (IQ) pre- and postoperation with assessment of the significance between and within both groups. Significance between groups-TA1: $p=0.03 ;^{*}$ TA2: $p=$ $0.01 ;{ }^{*}$ TA3: $p=0.003 .^{\dagger}$ Significance within groups-play group: $p=$ 0.04 ; $^{*}$ control group: $p=0.74$. ${ }^{*}$ Significance set at $p=0.05$. ${ }^{\dagger}$ Significance set at $p=0.01$. TA1 $=$ time point of assessment 1 day preoperation, TA2 = time point of assessment 2 days postoperation, TA3 = time point of assessment 9 days postoperation. 


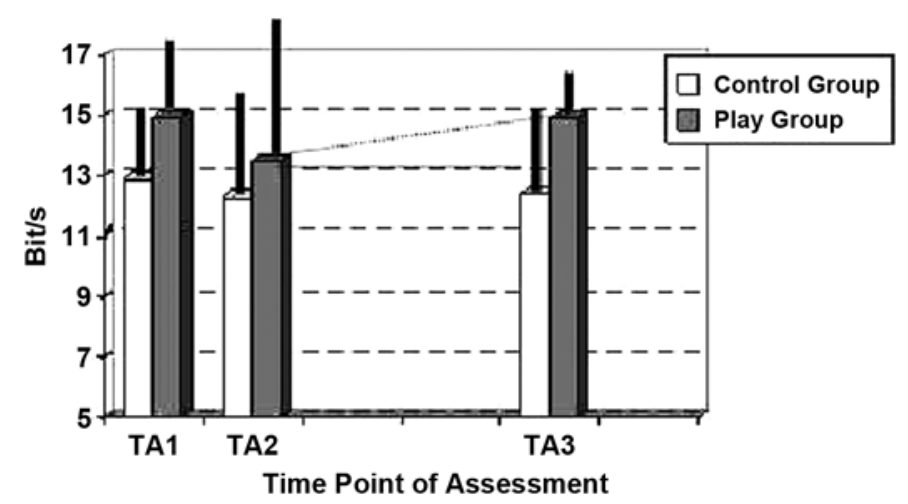

Figure 2.

Median and mean quartile of rate of information processing (bit/s) pre- and postoperation with assessment of significance of differences between and within both groups. Significance between groups-TA1: $p=0.13$; TA2: $p=0.09$; TA3: $p=0.03$. Significance within groupsplay group: $p=0.03 ;^{*}$ control group: $p=0.68$. ${ }^{*}$ Significance set at $p=$ 0.05 . TA1 $=$ time point of assessment 1 day preoperation, TA2 $=$ time point of assessment 2 days postoperation, TA3 = point of assessment 9 days postoperation.

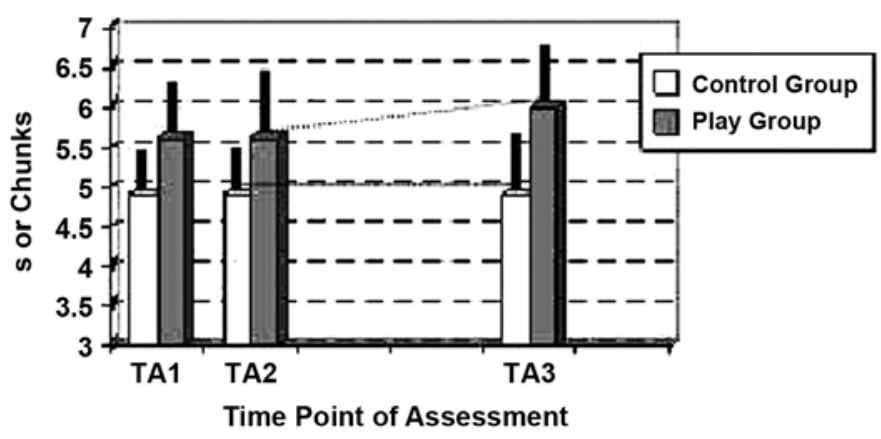

Figure 3.

Median and mean quartile of memory span (s or chunks [i.e., units in mind such as letters, digits, or one-syllable words]) pre- and postoperation with assessment of significance of differences between and within both groups. Significance between groups-TA1: $p=0.001{ }^{*}$ TA2: $p=$ $0.02{ }^{\dagger}$ TA3: $p=0.002{ }^{*}$ Significance within groups-play group: $p=$ 0.33 ; control group: $p=0.48$. * Significance set at $p=0.01$. ${ }^{\dagger}$ Significance set at $p=0.05$. TA $1=$ time point of assessment 1 day preoperation, TA2 = time point of assessment 2 days postoperation, TA3 = time point of assessment 9 days postoperation.

memory capacity because this magnitude is the base to determine IQ. To control different levels, we correlated the working memory capacity at TA2 ([TA3 - TA2] and [TA3 - TA1], $\rho=0.24[p=0.38])$ with the differences compared with TA1 $(\rho=0.22[p=0.43])$. According to these results, the patients with different mental efficiency had the same capacity for change.

\section{DISCUSSION}

During the course of the 7 postoperation days that the play group played daily, fluid IQ increased significantly from 99 to 106 (medians). The same was true for working memory capacity, as well as for rate of information processing. In contrast, the mental performance of the control group did not improve significantly during this period. Memory span did not change systematically in either group. At TA3, the play group was significantly superior to the control group in all cognitive tests. However, with the exception of rate of information processing, this superiority was already apparent at TA2 and even at TA1.

In Figures 1 and 2, the medians of the play group decreased more after surgery than that of the control group. This may be explained by the different distributions of the scores; in the medium range, the control group approached a normal distribution, whereas the values of the play group were scattered more individually. A slight change in the median thus causes a larger change in the values.

After controlling for the initial differences in cognitive performance of both groups, it is apparent that $D r$. Kawashima's Brain Training: How Old Is Your Brain? increased the rate of information processing but not the length of memory span. These two basic parameters of information processing determined the two other parameters investigated (working memory capacity and level of fluid intelligence). Thus, the two latter parameters also increased in correlation with the rate of information processing.

The advantage of an increase in the rate of information processing is that it increases fluid intelligence. Rueda et al. proved this by employing tests that postulated two distinct dimensions for the determination of the rate of information processing, namely complex and precise mental activity [10]. As shown in a number of studies, the mental parameters investigated correspond with success at school, work (including a correspondingly high income), and everyday life [10-13]. A further advantage of increased rate of information processing is that it accelerates and improves many processes of everyday life: faster reading, better comprehension of quickly spoken sentences, more rapid reactions in road traffic, etc. [14]. Therefore, patients who use VGs during prolonged hospitalization can reap subsequent benefits in their everyday life. 
Why did the length of memory span only negligibly respond to the use of specific VGs? Does Dr. Kawashima's Brain Training: How Old Is Your Brain? not train this parameter, or do the exercises have no effect? The basic parameter for memory span is not adequately exercised until the player trains with this VG continuously for prolonged sessions at advanced levels. We gave most of the patients sessions to increase the length of memory span no sooner than 3 or 4 days postoperation. The VG always started with exercises for improving the rate of information processing. Patients played the exercises for improving the memory span more rarely. This could explain the lack of effect on memory span within the relatively short period of only 7 days of training. Nevertheless, the statistical differences concerning the length of memory span between the play and control groups became more apparent between TA2 and TA3 (Table). Scanlon et al. and Klingberg et al. have empirically shown that the length of memory span and related parameters, such as working memory capacity and fluid intelligence, can be increased by exercises similar to those included in Dr. Kawashima's Brain Training: How Old Is Your Brain? [15-16].

When analyzing the differences in the effects of VGs on mental performance between the play and control groups at TA3, one should also consider that the evaluations at each TA took about 1 hour, which was longer than some of the VG sessions, and may therefore have their own training effects. For this reason, it can be assumed that the persons in the control group were more mentally activated than patients who were not involved in such studies. Thus, the differences we identified between the play and control groups may even be underestimated compared with unbiased conditions.

Can these results be generalized to different age groups and different levels of intelligence? Are the findings valid for both sexes and to the same extent? Within the play group, we correlated the differences in the basic parameters and the fluid IQ between TA2 and TA3 with age, sex, and basic levels of the premorbid and fluid intelligence. This allowed us to detect any association between the initial values of the parameters and changes during training. Sex and the basic level of premorbid intelligence were not associated with changes in cognitive performance. Therefore, these variables seem to be irrelevant for the training effects. However, there was a significant correlation with age. According to Spearman rank correlation, age correlated with the difference in length of memory span $(\rho=-0.69[p=0.005,2$-sided $])$ and with changes in fluid IQ ( $\rho=-0.56[p=0.03$, 2-sided)]. The correlations can be explained as follows: the older the patient, the less pronounced the increase in memory span and fluid IQ. The change in the rate of information processing, however, was not influenced by age.

Patients who achieved a high fluid IQ at TA2, thus demonstrating their high mental fitness, had a less pronounced increase in the rate of information processing than patients with a lower level of mental performance ( $\rho=-0.53$ ). Though this difference almost reached the level of significance ( $p=0.05$, 2-sided), we found no correlation with changes in memory span and fluid IQ.

The crystallized intelligence of the play group (median IQ 118) was significantly higher than that of the control group (median IQ 107) (Table). In agreement with these findings, Fischhof et al. reported that seniors with above-average IQ are able to play such VGs independently, whereas those with a lower mental performance had to be instructed and continually guided [17]. Overall, crystallized intelligence of our participants was generally relatively high. The IQ of our study participants distinctly exceeded the IQ levels of the general population, whose average IQ is 100.

The apparent difference in IQ between the play and control groups may be linked to age. As younger age groups are more familiar with VGs, their scores may benefit from a degree of openness and associated intelligence that may be required for a VG such as Dr. Kawashima's Brain Training: How Old Is Your Brain? [8].

The fact that the current cognitive performance decreased far below the level the patients had achieved at about the age of 20, which is indicated by the results in crystallized intelligence, suggests a lack of open-mindedness for new tasks. However, the level of fluid intelligence in the play group was preoperatively determined to be 104.5, while crystallized intelligence was 118 (median: compared to the normal values of persons 18-25 years old). In the control group, the fluid intelligence was 90 and the crystallized intelligence was 107 . Such a prominent decrease in the IQ of 68-year-old persons compared with 20 -year-old persons suggests an unfavorable way of life as far as mental performance is concerned.

Informing senior patients, in particular, of the results of this study, could motivate them to exercise with mentally activating VGs, thus improving their mental performance. This would provide benefits not only during hospitalization, including communication skills during that 
period, but also during subsequent reintegration into everyday life.

This study evaluated the value of VGs for the preservation of cognitive function in patients during prolonged hospitalization. This pilot study was not intended to evaluate the use of VGs in a large number of patients. The small number of patients must be considered a limitation of this study.

Nevertheless, playing VGs led to significant increases in central cognitive variables. With reference to the small samples, this indicates a relevant effect. On the other hand, some cognitive changes failed to reach statistical significance and the two samples could not be matched ideally in their cognitive level. However, according to theoretical connections between the administered variables here (rate of information processing, working memory capacity, and fluid intelligence) and compared with published research on partially overlapping aspects (rate of information processing [2,10], working memory capacity $[9,16]$, and fluid intelligence [9,14-15]), it seems that VGs can be beneficially applied to postsurgical rehabilitation. Before a practical expansion, the results of this study should be corroborated in larger populations.

\section{CONCLUSIONS}

Playing VGs developed for mental activation, such as Dr. Kawashima's Brain Training: How Old Is Your Brain?, may counteract the cognitive decay associated with prolonged hospitalization.

Exercises may be offered even to aged but mentally active patients previously unfamiliar with such VGs. They would benefit by retaining or even improving their mental fitness during hospitalization, and afterwards the exercises would enable them to cope more easily with the difficulties of everyday life.

Patients who are less mentally active need extra motivation, which could be provided by the feedback of the achieved profits. Persons with an already active mind and high mental performance, which correlates with crystallized IQ, will be able to use VGs by themselves after initial motivation. Other patients might need more individual support until they are unable to turn down assistance [17]. The increase in mental performance may allow some patients to become more independent and confident with the exercises. Further studies will determine whether these expectations can be fulfilled.

\section{ACKNOWLEDGMENTS}

\section{Author Contributions:}

Study concept and design: M. H. Brem, S. Lehrl. Acquisition of data: M. H. Brem, S. Lehrl, A. K. Rein, S. Massute, S. Schulz-Drost, H. J. Jacob, J. Gusinde.

Analysis and interpretation of data: M. H. Brem, S. Lehrl, A. K. Rein, S. Massute, S. Schulz-Drost, H. J. Jacob, J. Gusinde.

Drafting of manuscript: M. H. Brem, S. Lehrl, K. Gelse.

Critical revision of manuscript for important intellectual content:

M. H. Brem, S. Lehrl, K. Gelse, P. M. Schlechtweg, F. F. Hennig,

A. Olk, J. Gusinde.

Statistical analysis: S. Lehrl.

Obtained funding: M. H. Brem, S. Lehrl.

Administrative, technical, or material support: M. H. Brem, S. Lehrl, F. F. Hennig, J. Gusinde.

Study supervision: M. H. Brem, S. Lehrl.

Financial Disclosures: The authors have declared that no competing interests exist.

Funding/Support: This material was based on work supported by a grant from Euro RSCG ABC. The funding institution did not have any influence on the design and execution of the study, analysis and interpretation of data, or writing of the manuscript.

Institutional Review: We obtained informed consent from each patient. The ethics committee of Friedrich-Alexander Universität Erlangen-Nürnberg approved this study, which conforms to the Declaration of Helsinki.

Participant Follow-Up: The authors plan to inform participants of the publication of this study.

\section{REFERENCES}

1. Billig N, Ahmed SW, Kenmore PI. Approaches to senior care \#1. Hip fracture, depression, and cognitive impairment: A follow-up study. Orthop Rev. 1988;17(3):315-20. [PMID: 3174211$]$

2. Rosser JC Jr, Lynch PJ, Cuddihy L, Gentile DA, Klonsky J, Merrell R. The impact of video games on training surgeons in the 21st century. Arch Surg. 2007;142(2):181-86.

[PMID: 17309970]

DOI:10.1001/archsurg.142.2.181

3. Lehrl S, Triebig G, Fischer B. Multiple choice vocabulary test MWT as a valid and short test to estimate premorbid intelligence. Acta Neurol Scand. 1995;91(5):335-45.

[PMID: 7639062]

DOI:10.1111/j.1600-0404.1995.tb07018.x

4. Lehrl S, Fischer, B. The basic parameters of human information processing: Their role in the determination of intelligence. Person Individ Diff. 1988;9(5):883-96.

DOI:10.1016/0191-8869(88)90006-2

5. Pfenninger EG, Durieux ME, Himmelseher S. Cognitive impairment after small-dose ketamine isomers in comparison to equianalgesic racemic ketamine in human volunteers. 
JRRD, Volume 47, Number 9, 2010

Anesthesiology. 2002;96(2):357-66. [PMID: 11818769] DOI:10.1097/00000542-200202000-00022

6. Bullinger M, Kirchberger I, editor. Der SF-36-Fragebogen zum Gesundheitszustand-Handbuch für die deutschsprachige Frageogen-Version. Göttingen (Germany): Hogrefe; 1998.

7. Borkenau P, Ostendorf F, editor. NEO-Fünf-FaktorenInventar (NEO-FFI) nach Costa und McCrae. Göttingen (Germany): Hogrefe; 1993.

8. Lehrl S; Die P-R-O Skalen. GfG Gesellsschaft für Gehirntraining. Ebersberg (Germany): Vless Verlag; 2003.

9. Jaeggi SM, Buschkuehl M, Jonides J, Perrig WJ. Improving fluid intelligence with training on working memory. Proc Natl Acad Sci U S A. 2008;105(19):6829-33. [PMID: 18443283]

DOI:10.1073/pnas.0801268105

10. Rueda MR, Rothbart MK, McCandliss BD, Saccomanno L, Posner MI. Training, maturation, and genetic influences on the development of executive attention. Proc Natl Acad Sci U S A. 2005;102(41):14931-36. [PMID: 16192352] DOI:10.1073/pnas.0506897102

11. Brooks DW, Shell DF. Working memory, motivation, and teacher-initiated learning. J Sci Educ Technol. 2006;15(1): 17-30. DOI:10.1007/s10956-006-0353-0

12. Gottfredson LS. Intelligence: Is it the epidemiologists' elusive "fundamental cause" of social class inequalities in health? J Pers Soc Psychol. 2004;86(1):174-99.

[PMID: 14717635] DOI:10.1037/0022-3514.86.1.174

13. Kuncel NR, Hezlett SA, Ones DS. Academic performance, career potential, creativity, and job performance: Can one construct predict them all? J Pers Soc Psychol. 2004;86(1): 148-61. [PMID: 14717633] DOI:10.1037/0022-3514.86.1.148

14. Willis SL, Tennstedt SL, Marsiske M, Ball K, Elias J, Koepke KM, Morris JN, Rebok GW, Unverzagt FW, Staddard AM, Wright E; ACTIVE Study Group. Long-term effects of cognitive training on everyday functional outcomes in older adults. JAMA. 2006;296(23):2805-14.

[PMID: 17179457]

15. Scanlon M, Sarkar K, Drescher D. Using the Web for behavioral research and intervention: Evidence from cognitive training. Society for Neuroscience Conference; 2007 Nov 6; San Diego, CA.

16. Klingberg T, Fernell E, Olesen PJ, Johnson M, Gustafsson P, Dahlström K, Forssberg H, Westerberg H. Computerized training of working memory in children with ADHD-A randomized, controlled trial. J Am Acad Child Adolesc Psychiatry. 2005;44(2):177-86. [PMID: 15689731] DOI:10.1097/00004583-200502000-00010

17. Fischhof PK, Friedmann A, Moslinger-Gehmayr R. The influence of acquired intelligence on cerebral impairment in the elderly. Drugs Today (Barc). 2001;37(10):697-702. [PMID: 12738967]

DOI:10.1358/dot.2001.37.10.844216

Submitted for publication February 1, 2010. Accepted in revised form June 2, 2010.

This article and any supplementary material should be cited as follows:

Brem MH, Lehrl S, Rein AK, Massute S, Schulz-Drost S, Gelse K, Schlechtweg PM, Hennig FF, Olk A, Jacob HJ, Gusinde J. Stop of loss of cognitive performance during rehabilitation after total hip arthroplasty_-Prospective controlled study. J Rehabil Res Dev. 2010;47(9):891-98.

DOI:10.1682/JRRD.2010.01.0011

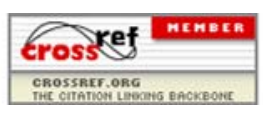

